SUPPORTING INFORMATION

For

\title{
Fast screening of coal fly ash with potential for rare earth element recovery by electron paramagnetic resonance spectroscopy
}

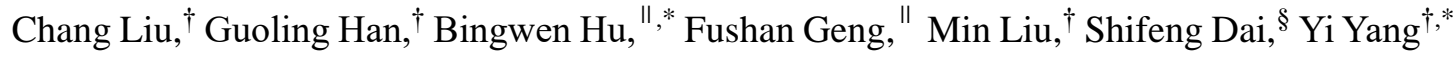

${ }^{\dagger}$ Key Laboratory of Geographic Information Science (Ministry of Education); School of

Geographical Sciences, East China Normal University, 500 Dongchuan Road, Shanghai 200241, China

"State Key Laboratory of Precision Spectroscopy, Shanghai Key Laboratory of Magnetic Resonance, School of Physics and Electronic Science, East China Normal University, Shanghai 200062, China;

${ }^{\S}$ State Key Laboratory of Coal Resources and Safe Mining, China University of Mining and Technology (Beijing), Beijing 100083, China

* Corresponding author:

Yi Yang. Phone: 86-21-54341196; Email: yyang@geo.ecnu.edu.cn; Bingwen Hu: bwhu@phy.ecnu.edu.cn 


\section{Contents:}

\begin{tabular}{|c|c|}
\hline Table S1. Detailed information of each designed method & Page S5 \\
\hline $\begin{array}{l}\text { Table S2. Concentration }(\mathrm{mg} / \mathrm{kg}) \text { and } \mathrm{RSD}(\%) \text { of each element in certified reference } \\
\text { material according to different methods }\end{array}$ & Page S6 \\
\hline $\begin{array}{l}\text { Table S3. Recovery (\%) of each element in certified reference material according to } \\
\text { different methods }\end{array}$ & Page S7 \\
\hline $\begin{array}{l}\text { Table S4. Recoveries (\%) and RSD (\%) of } \mathrm{Al} \text { and } \mathrm{Ca} \text { in certified reference material } \\
\text { according to Method } 2\end{array}$ & Page S8 \\
\hline Table S5. Microwave digestion program of Method 2 & Page S8 \\
\hline Table S6. EPR measurement parameter settings & Page S10 \\
\hline $\begin{array}{l}\text { Table S7. Mn (II) with different concentrations and their corresponding integrated } \\
\text { areas }\end{array}$ & Page S11 \\
\hline $\begin{array}{l}\text { Figure S1. Distribution pattern of REY in CFAs confirming the quality of the REY } \\
\text { analyzed in this study }\end{array}$ & Page S8 \\
\hline $\begin{array}{l}\text { Figure S2. EPR spectrums in foreign CFAs, including (A) } 3 \text { from the U.S. labeled } \\
\text { US-1, 2, 3, (B) } 2 \text { from Germany labeled Germany-1, } 2 \text { and (C) } 1 \text { from India labeled } \\
\text { India. }\end{array}$ & Page S9 \\
\hline Figure S3. EPR spectrum with six-fold resonances of trace $\mathrm{Mn}$ (II) in $\mathrm{CaO}$ powder & Page S10 \\
\hline $\begin{array}{l}\text { Figure S4. Calibration curve between } \mathrm{Mn} \text { (II) concentrations in } \mathrm{CaO} \text { powder and the } \\
\text { integrated area from magnetic field } 315 \text { to } 375 \mathrm{mT} \text { by EPR }\end{array}$ & Page S11 \\
\hline $\begin{array}{l}\text { Figure S5. Mn (II) concentrations based on EPR calibration showing significantly } \\
\text { negative correlation with } \mathrm{Ca}(\mathrm{A}) \text { and positive correlation with REY concentrations } \\
\text { (B) }\end{array}$ & Page S12 \\
\hline
\end{tabular}




\section{Sample digestion and determination}

All the reagents were provided by Sinopharm Chemical Reagent Co., Ltd. The specifications of $\mathrm{HClO}_{4}$ and $\mathrm{H}_{3} \mathrm{BO}_{3}$ were Guaranteed reagent (GR), $\mathrm{HBF}_{4}$ was Analytical reagent (AR) and the rest were Ultra-Metal-oxide-semiconduceopr (CMOS). The water used in this article was Milli-Q $\left(18.2 \mathrm{M} \Omega \cdot \mathrm{cm}^{-1}\right)$. In view of the potential danger related to $\mathrm{HClO}_{4}$, the equipped microwave digestion device (WX-8000, Kem, China) was designed to withstand high temperature and pressure.

To obtain an optimized method for measuring REY concentrations, 11 methods were tested and compared (Table S1). Certified reference material (Fine fly ash, CTA-FFA-1, Institute of Nuclear Chemistry and Technology, Poland) was applied, 3 blank and 6 reference material samples were tested in each designed method for data quality control and assurance.

Elements were measured by inductively coupled plasma mass spectrometry (ICP-MS, Perkin Elmer Nexion 1000, USA) in kinetic energy discrimination (KED) mode. Before ICPMS analysis, the ratio of $\mathrm{CeO}(156)$ / Ce (140) was kept below 0.025 to avoid the interferences of oxides, the index of In was kept above 100,000 to ensure sufficient signal strength. The instrument was calibrated by multi-elemental standard solutions with concentrations of $0.1 \mathrm{ppb}, 0.5 \mathrm{ppb}, 1 \mathrm{ppb}, 5 \mathrm{ppb}, 10 \mathrm{ppb}, 50 \mathrm{ppb}$ and $100 \mathrm{ppb}$. It was worth noting that when measuring major elements such as $\mathrm{Al}$ and $\mathrm{Ca}$, the concentrations of $\mathrm{Al}$ and $\mathrm{Ca}$ in standard solutions were 1 ppm, 2 ppm, 5 ppm, 10 ppm, 20 ppm, 50 ppm and 100 ppm. To ensure test accuracy and protect the detector, the count per second (cps) value of each major element was controlled below 1,000,000 through regulating electronic dilution factors. All the calibration curves for the elements were linear $\left(R^{2}>0.999, n=7\right)$ over the 
23

concentration range. After ICP-MS analysis, Method 2 was considered the most appropriate method for the digestion of CFA samples in the present study (Table S2-3). In addition, this method was also appropriate for $\mathrm{Al}$ and Ca determination (Table S4). In Method 2, the digestion program included two steps as shown in Table S5. After ICP-MS analysis, the average REY concentration in the present study was normalized to the Upper Continental

Crust value. ${ }^{1}$ As shown in Figure S1, the distribution pattern of REY was smooth and exhibited similar trend to previous studies carried out by Wang ${ }^{2}$ and $\mathrm{Li}^{3}{ }^{3}$ which reconfirmed the quality of the analytical REY data in this article. ${ }^{4}$ From the comparison of different digestion methods, it can be concluded that (1) temperature, (2) re-digested with $\mathrm{H}_{3} \mathrm{BO}_{3}$ and (3) the direct participation of $\mathrm{HClO}_{4}$ through the whole digestion process are critical factors. 
Table S1. Detailed information of each designed method

\begin{tabular}{|c|c|c|c|c|c|c|c|c|c|}
\hline Method & $\mathrm{HNO}_{3}$ & $\mathrm{HF}$ & $\mathrm{HClO}_{4}$ & $\mathrm{H}_{2} \mathrm{O}_{2}$ & $\begin{array}{l}\text { Chloronitric } \\
\text { acid }\end{array}$ & $\mathrm{HBF}_{4}$ & Step 1 & Step 2 & Step 3 \\
\hline 1 & $4 \mathrm{ml}$ & I & $0.5 \mathrm{ml}$ & I & I & $4 \mathrm{ml}$ & $220^{\circ} \mathrm{C}, 30 \mathrm{~min}$ & Diluted to $50 \mathrm{ml}$ with $2 \% \mathrm{HNO}_{3}$ & None \\
\hline 2 & $4 \mathrm{ml}$ & $2 \mathrm{ml}$ & $0.5 \mathrm{ml}$ & / & / & / & $220^{\circ} \mathrm{C}, 30 \mathrm{~min}$ & Diluted by $10 \mathrm{ml} \mathrm{H}_{3} \mathrm{BO}_{3}$ and re-digested at $180^{\circ} \mathrm{C}$ for $20 \mathrm{~min}$ & $\begin{array}{l}\text { Diluted to } 50 \mathrm{ml} \text { with } \\
2 \% \mathrm{HNO}_{3}\end{array}$ \\
\hline 3 & $4 \mathrm{ml}$ & $2 \mathrm{ml}$ & $0.5 \mathrm{ml}$ & I & I & / & $200^{\circ} \mathrm{C}, 30 \mathrm{~min}$ & Diluted by $10 \mathrm{ml} \mathrm{H}_{3} \mathrm{BO}_{3}$ and re-digested at $180^{\circ} \mathrm{C}$ for $20 \mathrm{~min}$ & $\begin{array}{l}\text { Diluted to } 50 \mathrm{ml} \text { with } \\
2 \% \mathrm{HNO}_{3}\end{array}$ \\
\hline 4 & $4 \mathrm{ml}$ & $2 \mathrm{ml}$ & $0.5 \mathrm{ml}$ & / & I & / & $200^{\circ} \mathrm{C}, 30 \mathrm{~min}$ & $\begin{array}{l}\text { Acid driven at } 180^{\circ} \mathrm{C} \text {, redissolved with } 1 \mathrm{ml} \mathrm{HNO}_{3} \text { and } 0.5 \mathrm{ml} \\
\mathrm{HClO}_{4} \text { and acid driven at } 180^{\circ} \mathrm{C} \text { again }\end{array}$ & $\begin{array}{l}\text { Diluted to } 50 \mathrm{ml} \text { with } \\
2 \% \mathrm{HNO}_{3}\end{array}$ \\
\hline 5 & $4 \mathrm{ml}$ & $2 \mathrm{ml}$ & I & $2 \mathrm{ml}$ & I & / & $200^{\circ} \mathrm{C}, 30 \mathrm{~min}$ & $\begin{array}{l}\text { Acid driven at } 180^{\circ} \mathrm{C} \text {, redissolved with } 1 \mathrm{ml} \mathrm{HNO}_{3} \text { and } 0.5 \mathrm{ml} \\
\mathrm{HClO}_{4} \text { and acid driven at } 180^{\circ} \mathrm{C} \text { again }\end{array}$ & $\begin{array}{l}\text { Diluted to } 50 \mathrm{ml} \text { with } \\
2 \% \mathrm{HNO}_{3}\end{array}$ \\
\hline 6 & $4 \mathrm{ml}$ & $2 \mathrm{ml}$ & I & I & I & l & $200^{\circ} \mathrm{C}, 30 \mathrm{~min}$ & $\begin{array}{l}\text { Acid driven at } 180^{\circ} \mathrm{C} \text {, redissolved with } 1 \mathrm{ml} \mathrm{HNO}_{3} \text { and } 0.5 \mathrm{ml} \\
\mathrm{HClO}_{4} \text { and acid driven at } 180^{\circ} \mathrm{C} \text { again }\end{array}$ & $\begin{array}{l}\text { Diluted to } 50 \mathrm{ml} \text { with } \\
2 \% \mathrm{HNO}_{3}\end{array}$ \\
\hline 7 & $4 \mathrm{ml}$ & $2 \mathrm{ml}$ & / & I & l & l & $200^{\circ} \mathrm{C}, 30 \mathrm{~min}$ & Acid dirven at $180^{\circ} \mathrm{C}$, adding $0.5 \mathrm{ml} \mathrm{HClO}_{4}$ in advance & $\begin{array}{l}\text { Diluted to } 50 \mathrm{ml} \text { with } \\
2 \% \mathrm{HNO}_{3}\end{array}$ \\
\hline 8 & / & $2 \mathrm{ml}$ & / & / & $6 \mathrm{ml}$ & / & $200^{\circ} \mathrm{C}, 30 \mathrm{~min}$ & Acid driven at $180^{\circ} \mathrm{C}$, adding $0.5 \mathrm{ml} \mathrm{HClO}_{4}$ in advance & $\begin{array}{l}\text { Diluted to } 50 \mathrm{ml} \text { with } \\
2 \% \mathrm{HNO}_{3}\end{array}$ \\
\hline 9 & l & $2 \mathrm{ml}$ & l & l & $6 \mathrm{ml}$ & / & $220^{\circ} \mathrm{C}, 30 \mathrm{~min}$ & Acid driven at $180^{\circ} \mathrm{C}$, adding $0.5 \mathrm{ml} \mathrm{HClO}_{4}$ in advance & $\begin{array}{l}\text { Diluted to } 50 \mathrm{ml} \text { with } \\
2 \% \mathrm{HNO}_{3}\end{array}$ \\
\hline 10 & / & $2 \mathrm{ml}$ & l & l & $6 \mathrm{ml}$ & l & $200^{\circ} \mathrm{C}, 30 \mathrm{~min}$ & Acid driven at $180^{\circ} \mathrm{C}$ & $\begin{array}{l}\text { Diluted to } 50 \mathrm{ml} \text { with } \\
2 \% \mathrm{HNO}_{3}\end{array}$ \\
\hline 11 & / & $2 \mathrm{ml}$ & / & / & $6 \mathrm{ml}$ & / & $220^{\circ} \mathrm{C}, 30 \mathrm{~min}$ & Acid driven at $180^{\circ} \mathrm{C}$ & $\begin{array}{l}\text { Diluted to } 50 \mathrm{ml} \text { with } \\
2 \% \mathrm{HNO}_{3}\end{array}$ \\
\hline
\end{tabular}


Table S2. Concentration $(\mathrm{mg} / \mathrm{kg})$ and RSD (\%) of each element in certified reference material according to different methods

\begin{tabular}{|c|c|c|c|c|c|c|c|c|c|c|c|c|c|c|}
\hline Method & & $\mathrm{Y}$ & $\mathrm{La}$ & $\mathrm{Ce}$ & $\mathrm{Nd}$ & $\mathrm{Sm}$ & $\mathrm{Eu}$ & $\mathrm{Gd}$ & $\mathrm{Tb}$ & Dy & $\mathrm{Er}$ & $\mathrm{Tm}$ & $\mathrm{Yb}$ & $\mathrm{Lu}$ \\
\hline \multirow{2}{*}{1} & Concentration & 9.17 & 12.7 & 28.4 & 17.3 & 3.54 & 0.73 & 3.30 & 0.41 & 2.93 & 1.65 & 0.20 & 1.38 & 0.18 \\
\hline & RSD & 2.07 & 140 & 7.58 & 12.1 & 11.3 & 262 & 6.97 & 395 & 9.22 & 6.67 & 380 & 7.25 & 33.3 \\
\hline \multirow[b]{2}{*}{2} & Concentration & 43.2 & 61.4 & 120 & 51.6 & 10.3 & 2.19 & 9.28 & 1.28 & 7.61 & 4.29 & 0.57 & 3.85 & 0.53 \\
\hline & RSD & 2.78 & 2.80 & 3.39 & 3.59 & 3.21 & 3.65 & 3.45 & 3.13 & 3.81 & 3.73 & 3.51 & 3.64 & 3.77 \\
\hline \multirow{2}{*}{3} & Concentration & 32.6 & 53.8 & 105 & 57.9 & 10.7 & 2.01 & 9.84 & 1.17 & 7.82 & 4.25 & 0.52 & 3.31 & 0.46 \\
\hline & RSD & 0.89 & 2.31 & 2.70 & 2.63 & 2.06 & 2.49 & 4.27 & 4.27 & 4.60 & 4.47 & 3.85 & 4.83 & 32.6 \\
\hline \multirow{2}{*}{4} & Concentration & 23.5 & 28.6 & 65.4 & 33.7 & 6.68 & 1.31 & 6.31 & 0.80 & 5.42 & 3.11 & 0.39 & 2.55 & 0.35 \\
\hline & RSD & 1.07 & 2.17 & 0.06 & 0.03 & 0.15 & 6.11 & 0.00 & 0.00 & 0.18 & 0.00 & 5.13 & 0.39 & 11.4 \\
\hline \multirow[b]{2}{*}{5} & Concentration & 23.5 & 30.3 & 62.4 & 25.9 & 4.99 & 1.08 & 4.46 & 0.67 & 3.74 & 2.14 & ND & 1.94 & 0.28 \\
\hline & RSD & 1.32 & 2.31 & 1.19 & 1.97 & 1.80 & 1.85 & 1.57 & 1.49 & 1.34 & 1.40 & l & 1.55 & 0.00 \\
\hline \multirow[b]{2}{*}{6} & Concentration & 41.3 & 42.2 & 89.4 & 41.2 & 8.40 & 1.84 & 7.54 & 1.08 & 6.39 & 3.70 & 0.31 & 3.34 & 0.46 \\
\hline & RSD & 0.85 & 0.90 & 2.04 & 0.66 & 0.84 & 1.09 & 0.66 & 0.93 & 0.63 & 0.81 & 9.68 & 0.60 & 0.00 \\
\hline \multirow{2}{*}{7} & Concentration & 9.89 & 4.57 & 14.6 & 6.21 & 1.55 & 0.35 & 1.54 & 0.26 & 1.71 & 1.10 & ND & 1.14 & 0.15 \\
\hline & RSD & 20.0 & 7.66 & 4.93 & 4.35 & 4.52 & 5.71 & 6.49 & 11.5 & 14.0 & 17.3 & I & 19.3 & 20.0 \\
\hline \multirow{2}{*}{8} & Concentration & 6.54 & 6.46 & 15.2 & 6.15 & 1.23 & 0.26 & 1.11 & 0.16 & 1.00 & 0.64 & ND & 0.63 & 0.08 \\
\hline & RSD & 11.0 & 10.8 & 10.9 & 11.1 & 10.6 & 11.5 & 10.8 & 12.5 & 11.0 & 9.38 & l & 7.94 & 0.00 \\
\hline \multirow{2}{*}{9} & Concentration & 36.5 & 55.7 & 111 & 45.0 & 8.83 & 1.93 & 8.95 & 1.25 & 6.72 & 3.86 & 0.30 & 3.51 & 0.51 \\
\hline & RSD & 0.82 & 1.20 & 1.09 & 1.38 & 1.13 & 1.04 & 0.89 & 0.80 & 1.79 & 2.07 & 23.3 & 1.42 & 1.96 \\
\hline \multirow{2}{*}{10} & Concentration & 4.84 & 4.18 & 12.8 & 4.94 & 1.09 & 0.24 & 0.99 & 0.14 & 0.95 & 0.62 & ND & 0.64 & 0.08 \\
\hline & RSD & 5.58 & 3.35 & 4.14 & 3.44 & 4.59 & 8.33 & 6.06 & 7.14 & 5.26 & 4.84 & I & 4.69 & 0.00 \\
\hline \multirow{2}{*}{11} & Concentration & 14.3 & 8.65 & 23.8 & 9.76 & 2.19 & 0.50 & 2.09 & 0.33 & 2.08 & 1.31 & ND & 1.28 & 0.18 \\
\hline & RSD & 18.9 & 15.6 & 13.9 & 9.22 & 3.20 & 6.00 & 6.22 & 9.09 & 12.9 & 16.0 & l & 18.8 & 22.2 \\
\hline
\end{tabular}

Notes: ND: Not detected; /: Not calculated 
Table S3. Recovery (\%) of each element in certified reference material according to different methods

\begin{tabular}{cccccccccccccc}
\hline Method & $\mathrm{Y}$ & $\mathrm{La}$ & $\mathrm{Ce}$ & $\mathrm{Nd}$ & $\mathrm{Sm}$ & $\mathrm{Eu}$ & $\mathrm{Gd}$ & $\mathrm{Tb}$ & $\mathrm{Dy}$ & $\mathrm{Er}$ & $\mathrm{Tm}$ & $\mathrm{Yb}$ & $\mathrm{Lu}$ \\
\hline 1 & 20.4 & 20.9 & 23.7 & 30.4 & 32.5 & 30.5 & 33.1 & 30.0 & 322 & 36.6 & 28.5 & 32.5 & 26.8 \\
2 & 96.0 & 101 & 99.7 & 90.8 & 94.3 & 91.7 & 92.8 & 92.7 & 83.7 & 94.8 & 80.4 & 90.8 & 80.3 \\
3 & 72.4 & 88.6 & 87.8 & 102 & 98.0 & 84.2 & 98.5 & 84.5 & 86.1 & 94.0 & 73.6 & 78.1 & 69.9 \\
4 & 52.2 & 47.1 & 54.5 & 59.3 & 61.3 & 54.7 & 63.1 & 58.1 & 59.7 & 68.9 & 55.7 & 60.1 & 53.2 \\
5 & 52.1 & 49.9 & 52.0 & 45.6 & 45.8 & 45.2 & 44.6 & 48.6 & 41.2 & 47.3 & $/$ & 45.7 & 42.5 \\
6 & 91.8 & 69.5 & 74.5 & 72.5 & 76.6 & 77.2 & 75.4 & 78.6 & 70.3 & 82.0 & 43.4 & 78.7 & 70.6 \\
7 & 22.0 & 7.53 & 12.2 & 10.9 & 14.3 & 14.8 & 15.4 & 18.6 & 18.8 & 24.4 & $/$ & 26.8 & 23.5 \\
8 & 14.5 & 10.6 & 12.7 & 10.8 & 11.3 & 10.7 & 11.1 & 11.2 & 11.0 & 14.2 & $/$ & 14.8 & 11.9 \\
9 & 81.1 & 91.8 & 92.9 & 79.1 & 81.0 & 80.7 & 89.5 & 90.4 & 73.9 & 85.4 & 42.6 & 82.7 & 77.8 \\
10 & 10.8 & 6.89 & 10.7 & 8.71 & 10.0 & 10.2 & 9.92 & 10.5 & 10.5 & 13.8 & $/$ & 15.2 & 12.2 \\
11 & 31.8 & 14.3 & 19.9 & 17.2 & 20.1 & 20.8 & 20.9 & 23.7 & 22.9 & 29.0 & $/$ & 30.1 & 27.8 \\
\hline
\end{tabular}

Note: /: Not calculated 
Table S4. Recoveries (\%) and RSD (\%) of Al and $\mathrm{Ca}$ in certified reference material according to Method 2

\begin{tabular}{ccc}
\hline Element & Recoveries & RSD \\
\hline $\mathrm{Al}$ & 95.5 & 2.65 \\
$\mathrm{Ca}$ & 94.5 & 2.90 \\
\hline
\end{tabular}

Table S5. Microwave digestion program of Method 2

\begin{tabular}{|c|c|c|c|c|c|}
\hline \multicolumn{3}{|c|}{ Step 1} & \multicolumn{3}{|c|}{ Step 2} \\
\hline${ }^{\circ} \mathrm{C}$ & $\mathrm{T}(\min )$ & $\mathrm{P}$ (atm) & ${ }^{\circ} \mathrm{C}$ & $\mathrm{T}(\min )$ & $\mathrm{P}(\mathrm{atm})$ \\
\hline 140 & 2 & 20 & 140 & 2 & 20 \\
\hline 160 & 2 & 25 & 160 & 2 & 25 \\
\hline 180 & 2 & 30 & 180 & 20 & 30 \\
\hline 200 & 2 & 35 & & & \\
\hline 220 & 30 & 40 & & & \\
\hline
\end{tabular}

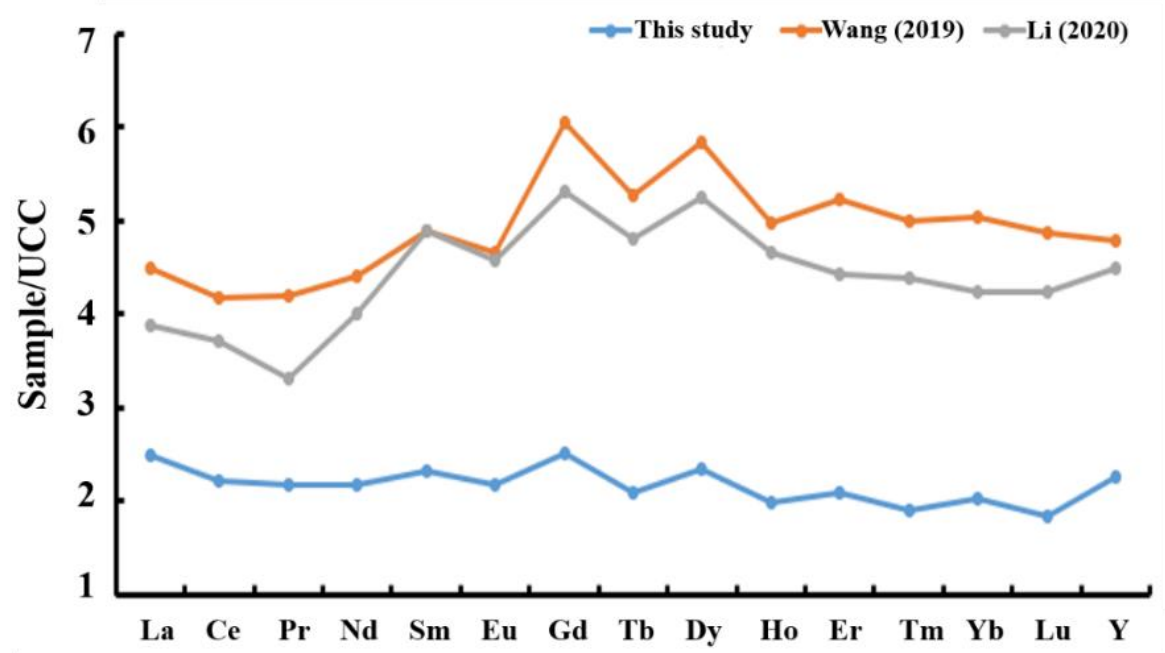

Figure S1. Distribution pattern of REY in CFAs confirming the quality of the REY analyzed in this study 

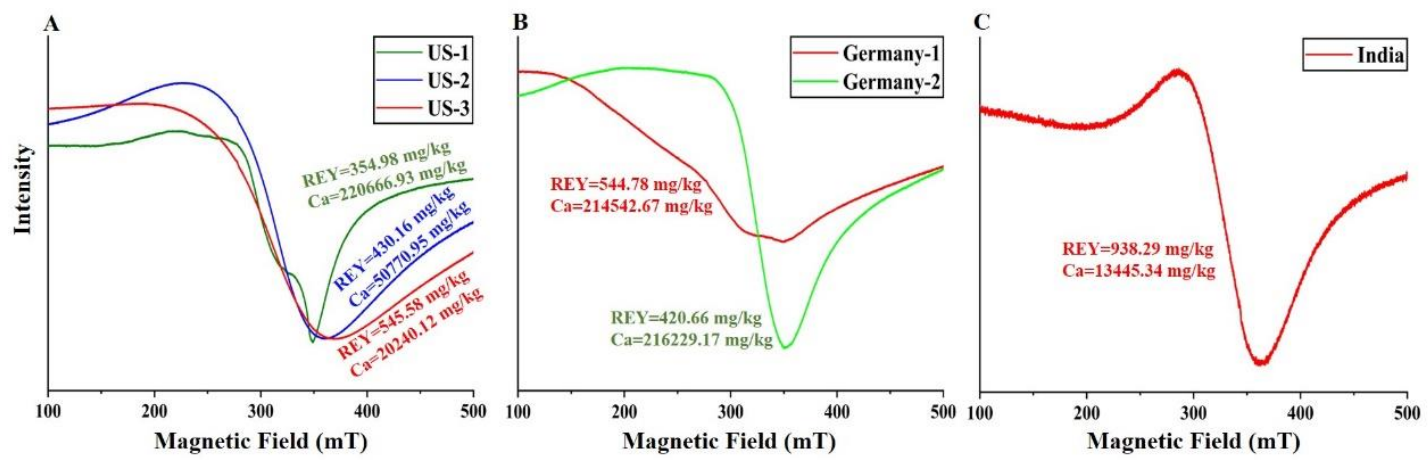

Figure S2. EPR spectrums in foreign CFAs, including (A) 3 from the U.S. labeled US-1, 2, 3, (B) 2 from Germany labeled Germany-1, 2 and (C) 1 from India labeled India. 


\section{Electron paramagnetic resonance (EPR) measurement}

$\mathrm{CaO}$ powder (provided by Titan Scientific Co., Ltd, $\mathrm{AR}, \geq 98 \%$ ) was applied as reference material in the present study. After measured the reference material by EPR (measurement parameter settings are shown in Table S6), six-fold resonances appeared as shown in Figure S2, locating from magnetic field 315-375 mT, with hyperfine coupling constant $A=9 \mathrm{mT}$. Mechanistically, the six-fold resonances are typical signals of Mn (II) in EPR measurement owing to the electronic configuration of $\mathrm{Mn}$ (II) $\left(3 d^{5} 4 s^{2}, I=5 / 2,2 I+1=6\right)$, and the six-fold resonances ought to be observed within the specific magnetic field range with a hyperfine coupling constant $A=8-10 \mathrm{mT}^{5,6}$

Table S6. EPR measurement parameter settings

\begin{tabular}{ccc}
\hline settings & parameters & value \\
\hline Magnetic Field & central magnetic field & $3480 \mathrm{G}$ \\
& sweep time & $100 \mathrm{~s}$ \\
& sweep width & $5000 \mathrm{G}$ \\
Signal Channel & receiver gain & 2 \\
Microwave & modulation amplitude & $30 \mathrm{~dB}$ \\
& microwave power & $2 \mathrm{G}$ \\
& Microwave attenuation & $100 \mathrm{mv}$ \\
& resolution & $30 \mathrm{~dB}$ \\
& & $25000 \mathrm{points}$
\end{tabular}

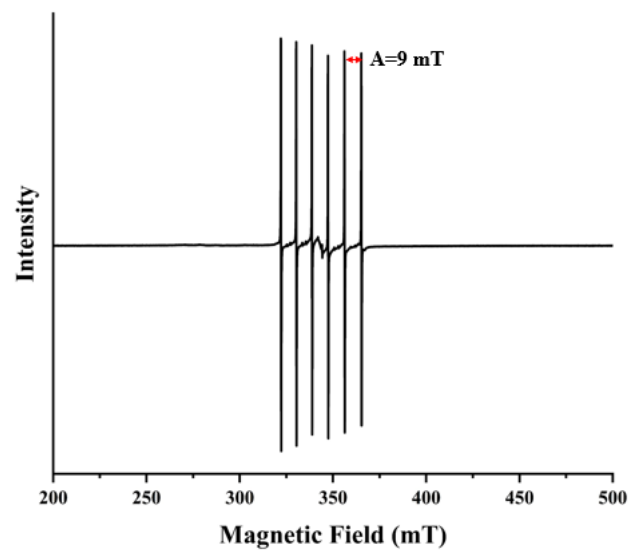

Figure S3. EPR spectrum with six-fold resonances of trace $\mathrm{Mn}$ (II) in $\mathrm{CaO}$ powder 
To reveal the accuracy of EPR in measuring Mn (II) concentrations in a certain range, a calibration curve was set up between the concentrations of Mn (II) and the corresponding integrated areas of the six-fold resonances according to EPR. Mn (II) concentration in the reference $\mathrm{CaO}$ was $28.58 \mathrm{mg} / \mathrm{kg}$ based on ICP-MS analysis. Five reference materials were solid-diluted by $\mathrm{SiO}_{2}$ powder ( provided by Sinopharm Chemical Reagent Co., Ltd, AR, $\geq 95 \%$ ) by $2,5,10,15$ and 20 times (Table S7), respectively, and then measured by EPR. After subtracting the background of $\mathrm{SiO}_{2}$ powder, it can be found the calibration curve was linear $\left(\mathrm{R}^{2}=0.999\right)$ over the concentration range of $\mathrm{Mn}$ (II) within the specific magnetic field range from 315 to $375 \mathrm{mT}$ (Figure S4).

Table S7. Mn (II) with different concentrations and their corresponding integrated areas

\begin{tabular}{ccc}
\hline Dilution factor & $\begin{array}{c}\text { Concentration of } \\
\text { Mn (II) (mg/kg) }\end{array}$ & $\begin{array}{c}\text { Integrated area } \\
\text { (Magnetic field 315-375 mT) }\end{array}$ \\
\hline 0 & 28.58 & 10921220.66 \\
2 & 14.29 & 5026837.51 \\
5 & 5.72 & 1573603.13 \\
10 & 2.86 & 645875.97 \\
15 & 1.91 & 321126.84 \\
20 & 1.43 & 214638.41 \\
\hline
\end{tabular}

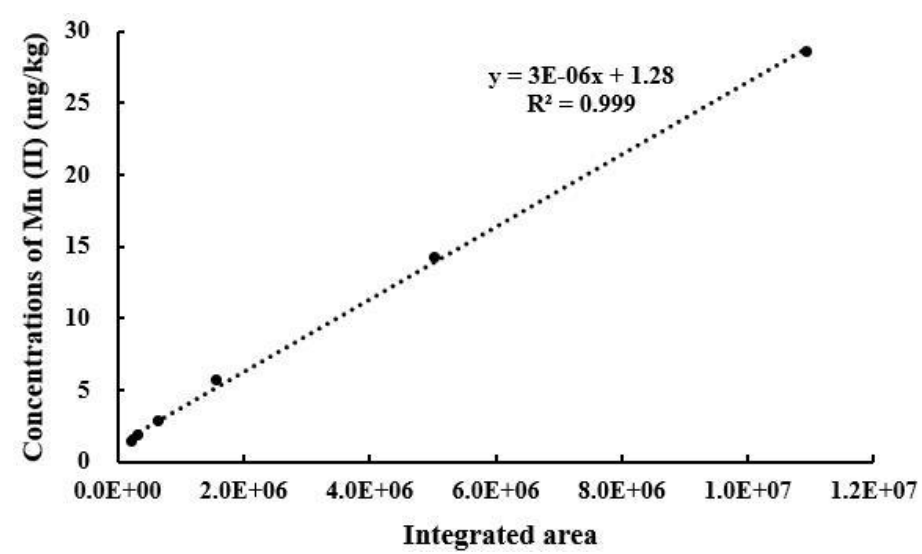

Figure S4. Calibration curve between $\mathrm{Mn}$ (II) concentrations in $\mathrm{CaO}$ powder and the integrated area from magnetic field 315 to $375 \mathrm{mT}$ by EPR 

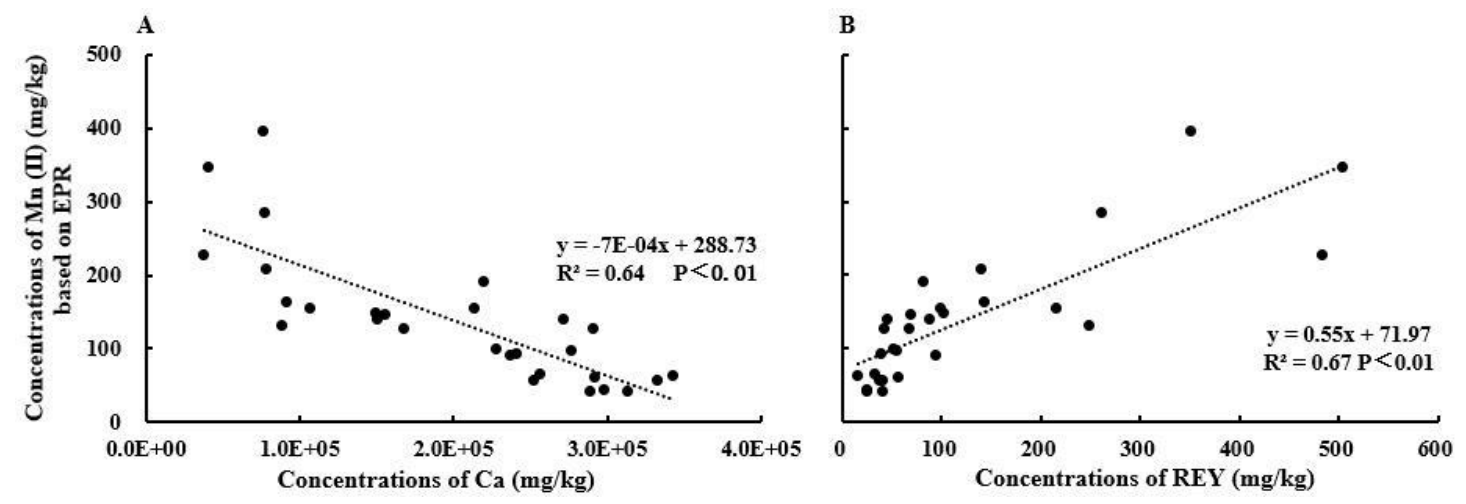

Figure S5. Mn (II) concentrations based on EPR calibration showing significantly negative correlation with $\mathrm{Ca}(\mathrm{A})$ and positive correlation with REY concentrations (B) 


\section{REFERENCES}

1. Taylor, S.R.; Mclennan, S.M. The geochemical evolution of the continental crust. Rev. Geophys. 1995, 33 (2), 241-265.

2. Wang, Z.; Dai, S.; Zou, J.; French, D.; Graham, I. T. Rare earth elements and yttrium in coal ash from the Luzhou power plant in Sichuan, southwest China: concentration, characterization and optimized extraction. Int. J. Coal Geol. 2019, 203, 1-14.

3. Li, Z.; Li, X.; Zhang, L.; Li, S.; Chen, J.; Feng, X.; Zhao, D.; Wang, Q.; Gao, Z.; Xiong, B. Partitioning of rare earth elements and yttrium (REY) in five coal-fired power plants in Guizhou, southwest China. J. Rare Earths. 2020, 38 (11), 1257-1264.

4. Dai, S.; Graham, I.T.; Ward, C.R. A review of anomalous rare earth elements and yttrium in coal. Int. J. Coal Geol. 2016, 159, 82-95.

5. Rakhmatullin, R.M.; Semashko, V.V.; Korableva, S.L.; Kiiamov, A. G.; Rodionov, A. A.; Tschaggelar, R.; van Bokhoven, J. A.; Paun, C. EPR study of ceria nanoparticles containing different concentration of $\mathrm{Ce}^{3+}$ ions. Mater. Chem. Phys. 2018, 219, 251-257.

6. McBride, M.B. On the natural Mn (II) EPR signal of SWy-1 montmorillonite. Clays Clay Miner. 1995, 43 (3), 383-384. 